\title{
Special optical fiber design to reduce reflection peak distortion of a FBG embedded in inhomogeneous material
}

\author{
Lun-Kai Cheng $^{*}$, Peter Toet $^{\mathrm{a}}$, Jan de Vreugd ${ }^{\mathrm{a}}$, Remco Nieuwland ${ }^{\mathrm{a}}$, \\ Ming-Leung Vincent Tse ${ }^{b}$, Hwayaw Tam ${ }^{b}$ \\ ${ }^{a}$ TNO, Stieltjesweg 1, 2628 CK Delft, The Netherlands; \\ ${ }^{\mathrm{b}}$ Photonics Research Centre, the Hong Kong Polytechnic University, Hong Kong
}

\begin{abstract}
During the last decades, the use of optical fiber for sensing applications has gained increasing acceptance because of its unique properties of being intrinsically safe, unsusceptible to EMI, potentially lightweight and having a large operational temperature range. Among the different Fiber Optic sensor types, Fiber Bragg Grating (FBG) is most widely used for its unique multiplexing potential and the possibility of embedding in composite material for Structural Health Monitoring. When the fiber is embedded in an inhomogeneous environment, typically a material composed of filler and base material of different stiffness, local stiff material will generate extra lateral load to the fiber. Via the Poisson effect, this will be converted to a local axial strain. The narrow and sharp peak in the reflection spectrum of an FBG sensor relies on the constant periodicity of the grating. An inhomogeneous axial strain distribution will result in distortion or broadening of the FBG reflection spectrum. For the FBG strain sensitivity of about $1.2 \mathrm{pm} / \mu \varepsilon$, the spectral distortion can be disastrous for strain measurements. A fiber design to tackle this critical problem is presented. Finite Element Modeling is performed to demonstrate the effectiveness of the solution. Modeling with different configurations has been performed to verify the influence of the design. The deformation of the core in the special fiber depends on the design. For a particular configuration, the core deformation in the axial direction is calculated to be a factor of 10 lower than that of a standard fiber. The first prototype fiber samples were drawn and the manufacturing of FBG in this special fiber using the phase mask method was demonstrated successfully.
\end{abstract}

Keywords: fiber optic sensor, Fiber Bragg Grating, composite, high-temperature, structural health monitoring

\section{INTRODUCTION}

In the last decades, Fiber Optic (FO) sensor has gained increasing acceptance in different applications. Among the different FO sensor types, Fiber Bragg Grating (FBG) is most widely used due to its unique multiplexing potential. FBG is a periodical modulation of the refractive index in an optical fiber. The reflection wavelength of an FBG depends on the period of the refractive index modulation. This is described by the Bragg's law: $\lambda_{B}=2 N_{\text {eff }} \Lambda$, where $\lambda_{B}$ is the center wavelength of the reflected light, $N_{\text {eff }}$ is the effective refractive index of the optical fiber and $\Lambda$ is the period of the modulation. Any physical parameters which affect either $N_{\text {eff }}$ or $\Lambda$ will cause a change in $\lambda_{\mathrm{B}}$. FBG sensors are mostly used for strain $(\varepsilon)$ or temperature (T) measurements. The coefficient $\Delta \lambda_{\mathrm{B}} / \Delta \varepsilon$ and $\Delta \lambda_{\mathrm{B}} / \Delta \mathrm{T}$ are $\sim 1.2 \mathrm{pm} / \mu \varepsilon$ and $\sim 10 \mathrm{pm} /{ }^{\circ} \mathrm{C}$ respectively for standard optical fiber operating in $1550 \mathrm{~nm}$ wavelength window.

The shape of the reflection spectrum with center wavelength $\lambda_{B}$ depends on the length $\mathrm{L}$ of the FBG and $\Delta \mathrm{n}$, the modulation in the refractive index in the fiber. The spectrum can be modelled accurately using Couple Mode Theory but a simple representation of the spectrum can be calculated from the Fourier transformation of the refractive index modulation function $\mathrm{n}(\mathrm{z})$.

$$
n(z)=n_{\text {core }}+\Delta n\left[1+\cos \left(2 \pi \frac{Z}{\Lambda}\right)\right]
$$

*lun.cheng@tno.nl; phone +31 888666 384; www.tno.nl 
The FWHM of the reflection peak $\Delta \lambda$ and the maximum reflection are determined by the FBG length $\mathrm{L}$ and $\Delta \mathrm{n}$. In general, the longer the FBG length $\mathrm{L}$ is, the smaller $\Delta \lambda$ will be and the better the shift in the center wavelength $\lambda_{\mathrm{B}}$ can be detected. FBGs with $\Delta \lambda$ down to $0.04 \mathrm{~nm}$ are commercially available. Using this type of small bandwidth FBG, we demonstrated a wavelength noise level of $\sim 0.03 \mathrm{fm} / \sqrt{ } \mathrm{Hz}$. This corresponds to a strain noise level of $\sim 0.025 \mathrm{n \varepsilon} / \sqrt{\mathrm{Hz}}$. $\underline{F o r}$ the development of a high sensitive FBG sensor system, a small and constant $\Delta \lambda$ and hence, an uniform periodicity of the entire FBG is required.

An important advantage of FBG sensors is that each FBG sensor can be designed to have a different Bragg wavelength to reflect. This enables the application of FBG sensors in an array configuration by simple multiplexing in the wavelength domain (see Figure 1).

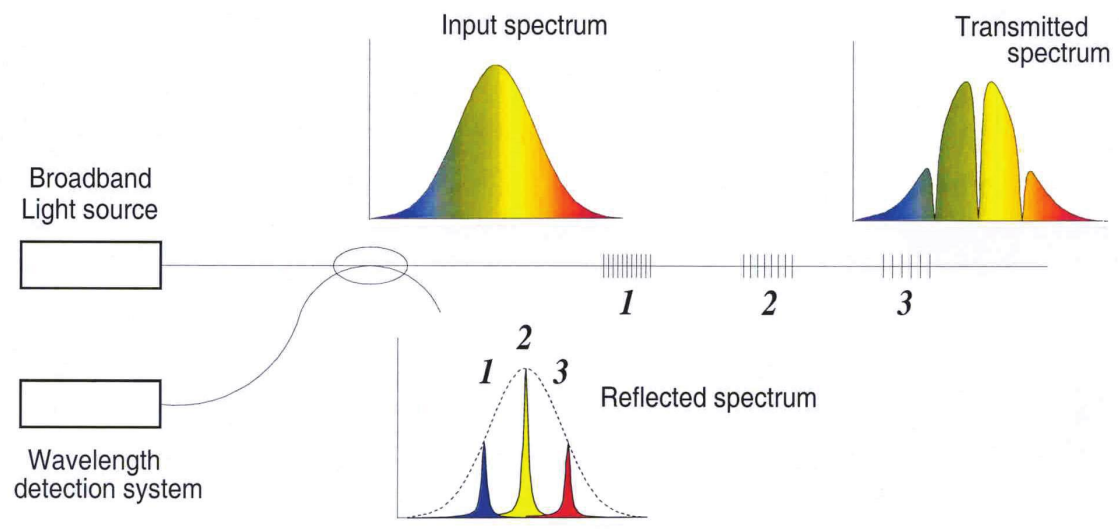

Figure 1. Basic configuration of an FBG sensor array. Each FBG reflects a different part of the input spectrum and can be identified by it's center wavelength.

Other advantages of FBG sensors are intrinsically safe, unsusceptible to EMI, lightweight and a large operational temperature range. When using FBG in high temperature environments, e.g. fiber optic sensing for Space or Oil\&Gas applications $[1,2,3]$, suitable high temperature adhesives have to be selected, and optimized gluing procedures have to be developed. High temperature adhesives can be used up to $1500{ }^{\circ} \mathrm{C}$ and are based on a two-components system with hard ceramics fillers. This type of 2-components adhesives will introduce inhomogeneous mechanical lateral load to the FBG due to the difference in stiffness between the base material and the filler. The stiff filler will generate locally higher mechanical stress to the fiber. Via the Poisson effect, this will be converted to locally a higher axial strain. This results in an inhomogeneous axial strain distribution along the FBG and hence a variation in the periodicity in Eq. 1 . The narrow and sharp peak in the reflection spectrum of an FBG sensor relies on the constant periodicity of the grating. Due to the random nature of the inhomogeneous axial strain, this results in an unpredictable distortion/broadening of the FBG reflection spectrum which is fatal for high accuracy strain measurement with FBG sensor.

\subsection{Example of spectral distortion/broadening}

An example of spectral distortion is shown in Fig. 2. Even for an FBG glued on a metal plate with a single-component adhesive, spectral distortion due to an improper gluing process is observed after a baking process up to $\sim 300{ }^{\circ} \mathrm{C}$. During the baking process, the temperature was increased slowly from $20{ }^{\circ} \mathrm{C}$ to $\sim 300{ }^{\circ} \mathrm{C}$ and returned slowly to $20{ }^{\circ} \mathrm{C}$. The spectrum of the FBG at the beginning of the experiment $\left(@ 20^{\circ} \mathrm{C}\right.$ ) has a single sharp peak with $\Delta \lambda=\sim 0.12 \mathrm{~nm}$ (solid line in Fig. 2). The spectra at different temperatures during the cooling down demonstrate spectral distortion/broadening (dashed lines in Fig. 2). 


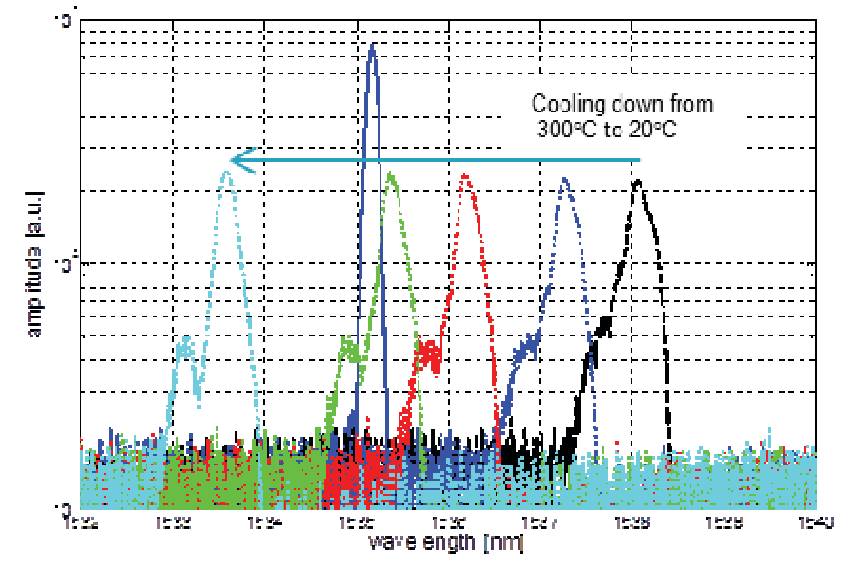

Figure 2. Spectral broadening/distortion of an FBG glued with a single-component high-temperature adhesive. Solid line: FBG spectrum at the start of baking process. Dashed lines: development of the spectrum during cooling down from $\sim 300^{\circ} \mathrm{C}$ to $\sim 20^{\circ} \mathrm{C}$.

\subsection{Spectral distortion for FBG embedded in composite}

Another situation of inhomogeneous load to the FBG is when the FBG is embedded in a composite material. For the development and manufacturing of large, special structures, weight reduction is becoming an increasingly critical issue. This weight reduction can be realized by replacing metal parts with composite parts for its superior strength-to-weight ratios. Current wind turbine blades are all made of composites, and in the commercial aircraft industry significant parts of newly developed aircraft such as the Boeing 787 and the Airbus A380 are also made of composite materials. The most important failure modes of composite materials are impact by an object, delamination and de-bonding. These failure modes can be monitored by FBG strain sensors at appropriate locations in the structures. Defects in the structure can be noticed at an early stage. This is in particular of interest for Prognostic Health Monitoring (PHM) of aerospace structures. By recording and analyzing the output of the FBG sensors in a PHM system, the condition of the structure can be determined. Based on some intelligent modeling, the maintenance schedule can be optimized to reduce the operational cost. Embedding the FBG in the composite structure has the added benefit of protecting the fiber and increasing its lifetime. In the past few years, the application of composite construction has also been introduced in the Oil \& Gas industry. Due to the inhomogeneity of these composite materials, embedding standard FBGs will lead to extreme distortions of the FBG reflection spectrum [4, 5], as discussed above. Spectral broadening even up to a few nm's is demonstrated $[6,7]$. Extra mechanical packaging of the fiber to isolate the fiber mechanically is not a workable solution for composite structure because the size of the sensor/fiber has to be kept as small as possible to minimize the effect on the mechanical strength of the composite structure.

\section{BASIC CONCEPT OF SPECIAL FIBER}

To tackle the problem described above, a special fiber design is invented. In this paper, the development of this fiber design to solve the problem of lateral load induced spectral distortion is presented. This solution can be used for FBG embedded in composite and FBG glued in two-components adhesives for high temperature applications. FBG manufactured with a special process, i.e. Chemical Composition Gratings (CCG) [8] can be used for operating temperature up to $900^{\circ} \mathrm{C}$. This type of FBG is recently commercially available [9] and enables many new applications of FBG sensor in high temperature environments.

By incorporating channels with a special shape along the fiber, the lateral load induced (local) strain/stress in the core of the fiber can be reduced. The proposed fiber concept consists of an inner part (including the core of the optical fiber) and an outer part. The two parts are connected by mechanical structures (connection beams) along the fiber. Air channels are between the mechanical structures. The shape of mechanical structures is designed to transfer radial compression of the fiber to a rotation, instead of compression of the fiber core (see Figure 3). 


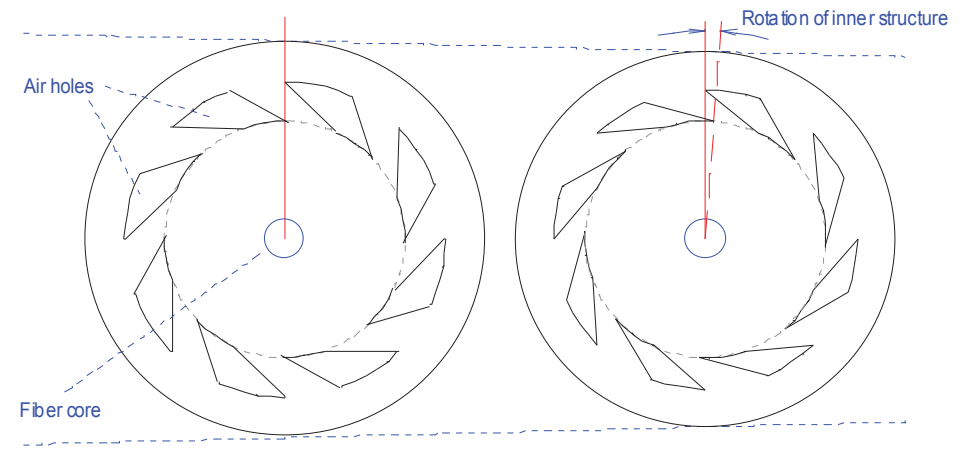

Figure 3. Design of the proposed fiber concept with 7 connection beams. Radial load will cause a rotation of the inner part with the fiber core.

When the fiber is stretched along the axial direction, the connection beams will transfer the axial strain from the outer part to the inner part where the FBG is located. The FBG will keep a constant grating period under both lateral and axial load, and the shape of the FBG reflection spectrum is remained. A patent is pending for this solution (WO2013112051).

The number, location and shape of the mechanical connection beams for linking the inner and outer parts determine the effectiveness of this solution. These parameters have to be selected for the optimization of the mechanical properties and/or the manufacturability of the fiber/FBG.

\section{FEM RESULTS}

Finite Element Modeling (FEM) was performed to compare the response between the proposed fiber concept and other concepts including the standard solid silica fiber and an air-hole fiber. For the modeling, the proposed concept with 3 connection beams is used to demonstrate the effect.

The first comparison is between the proposed concept and a standard solid core fiber. For the FEM modeling, both fibers are located between 2 load plates with 4 cylindrical structures as shown in Figure 4(a). The cylindrical structures have the same radius as the fibers. The deformation of the core is calculated for a displacement of $1 \mu \mathrm{m}$ of the upper load plate in the y-direction (arrow in Figure 4(a)) while x-direction is the axial direction of the fiber. The lateral load induced axial deformation is represented by the $\mathrm{x}$-displacement $\Delta \mathrm{x}$ of the center of the fiber. This depends on the distance to the load location $\mathrm{x}_{\text {load. }}$ (see Fig. 4(b) and 4(c)).

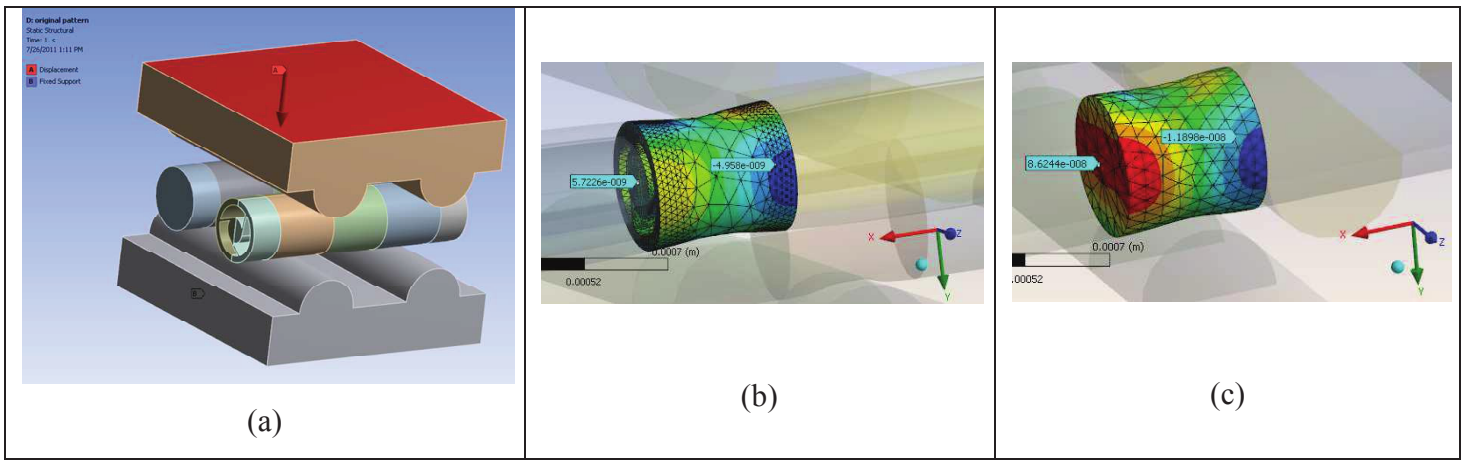

Figure 4. (a) Configuration for the FEM analysis for load in the y-direction. Calculation of x-displacement of the core in the proposed fiber (b) and the standard fiber (c). 
The $\mathrm{x}$-displacement $\Delta \mathrm{x}$ at a distance of $10 \mu \mathrm{m}$ from $\mathrm{x}_{\text {load }}$ for both fibers are calculated and compared. For the standard solid core fiber, $\Delta \mathrm{x}$ is $11.6 \mathrm{~nm}$. For the proposed fiber with 3 connection beams, this is reduced by a factor of 18 to $0.6 \mathrm{~nm}$ (See Table 1).

To demonstrate the effect of the shape of the connection beam, FEM analysis is also performed with a fiber design with 75 circular air holes along the fiber. The number and size of air holes are selected to have the same surface of open area as in the proposed fiber design in Fig. 4(a).

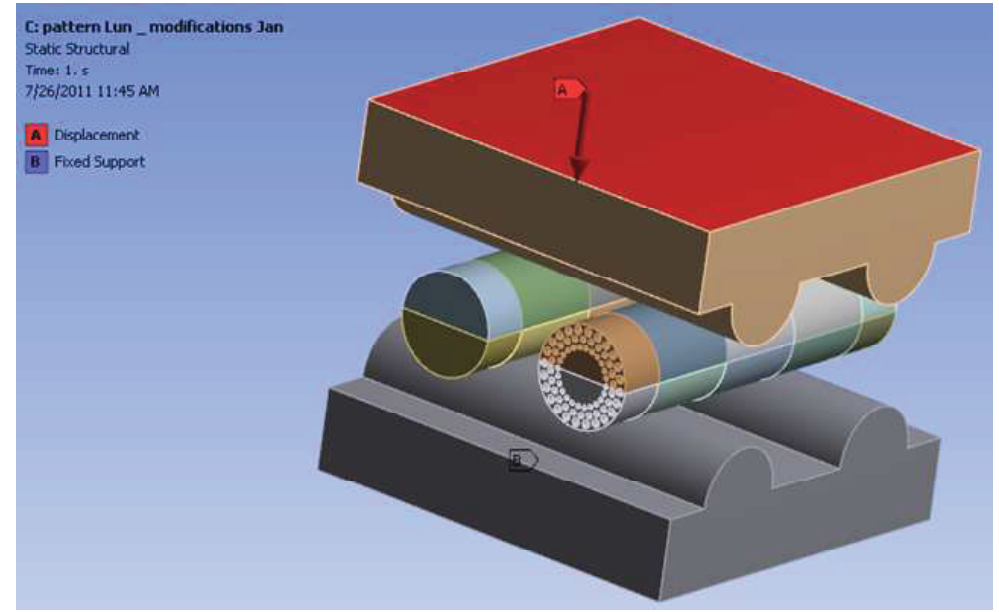

Figure 5. FEM configuration for the comparison between air-hole fiber and standard solid core fiber.

In comparison to the standard solid core fiber, the $\mathrm{x}$-displacement at a distance of $10 \mu \mathrm{m}$ from $\mathrm{x}_{\mathrm{load}}$ is reduced by about a factor of 2 only $(6.5 \mathrm{~nm}$ versus $11.6 \mathrm{~nm})$. The results are summarized in Table 1.

Table 1. $\mathrm{x}$-displacement in different fiber designed as a result of $1 \mu \mathrm{m}$ y-displacement.

\begin{tabular}{|l|c|c|}
\hline \multicolumn{1}{|c|}{ Fiber design } & x-displacement $@ \mathbf{1 0} \boldsymbol{\mu m}$ from $\mathbf{x}_{\text {load }}$ & Improvement \\
\hline Standard solid fiber & $11.6 \mathrm{~nm}$ & - \\
\hline $\begin{array}{l}\text { Proposed special design fiber } \\
\text { with 3 connection beams }\end{array}$ & $0.6 \mathrm{~nm}$ & $\sim 18 \mathrm{x}$ \\
\hline $\begin{array}{l}\text { Air-hole fiber with 75 air-holes } \\
\text { along the fiber }\end{array}$ & $6.5 \mathrm{~nm}$ & $\sim 2 \mathrm{x}$ \\
\hline
\end{tabular}

The deformation of the core in the special fiber is calculated to be a factor of 10 lower than that in a standard fiber. Calculations with other configurations of beams have also been performed to optimize the design.

\section{MANUFACTURING OF PROPOSED FIBER SAMPLE INCLUDING FBG}

Two batches the proposed fiber concept were manufactured by the Hong Kong Polytechnic University using the stacking method. The first batch has 4 connection beams (Fig. 6(a)) and about 100m of this fiber is drawn. The shape of the structure at different part of the fiber is inspected and found to be consistent. After drawing of the fiber, FBGs were fabricated successfully in the fiber after hydrogen loading. The phase mask method with a 193-nm laser was used to 
inscribe the grating. The reflection wavelength of the FBG was measured to be $1550.5 \mathrm{~nm}$. The spectrum is shown in Figure 6(b). For the second batch, a design with 5 connection beams is used to demonstrate the effect of the design configuration. FBGs were also fabricated in the second batch fiber. Tests of the FBGs under lateral load to verify the desire properties are scheduled.

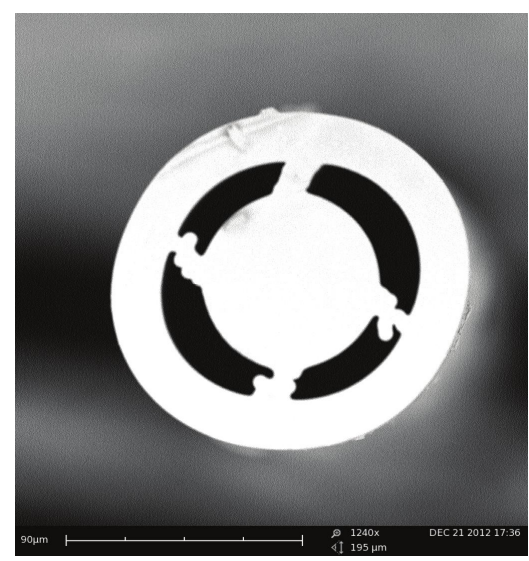

(a)

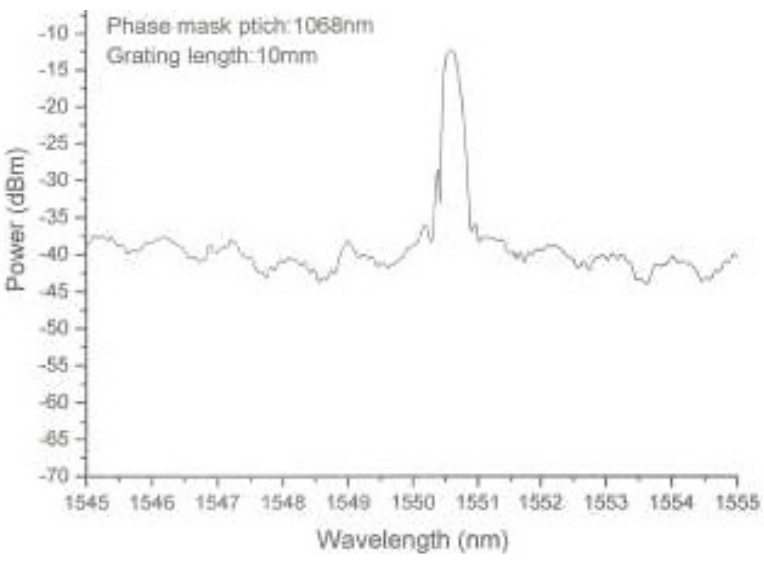

(b)

Figure 6. (a) Cross section of a manufactured fiber with 4 connection beams based on the design as proposed in this paper. (b) Reflection spectrum of an FBG manufactured in a sample of the first batch fiber with the special design.

\section{REFERENCES}

[1] Yamate, T., et al., "Thermally Insensitive Pressure Measurements up to 300 degree C using Fiber Bragg Gratings Written onto Side Hole Single Mode Fiber,” Proceedings of SPIE, Vol. 4185, 628-631 (2000).

[2] Yamate, T., "Fiber Optic Sensors for the Exploration of oil and Gas", Proceedings of SPIE, Vol. 7004, 700438 (2008).

[3] http://smartfibres.com/docs/SmartFlow_2p.pdf

[4] Qi, B., et al., "Response of an embedded fibre Bragg grating to thermal and mechanical loading in a composite laminate", Materials Forum, Vol. 27, 93-100 (2004).

[5] Hao, J., et al., "Realization of an Embedded Fiber Bragg Grating Based Pressure Sensor in Fiber Reinforced Composites: Embedding Techniques and Performance Characteristics", Proceedings of SPIE, Vol. 5279, 111125 (2004).

[6] Webb, S., et al., "Wavelength hopping due to spectral distortion in dynamic fiber Bragg grating sensor measurements", Meas. Sci. Techno., Vol. 22, 065301 (2011).

[7] Okabe, Y., et al., "Effect of fiber coating on crack detection in carbon fiber reinforced plastic composites using fiber Bragg grating sensors", Smart Mater. Struct., Vol. 11, 892-898 (2002).

[8] https://www.acreo.se/groups/fiber-bragg-gratings-fbg-laboratory

[9] http://www.specialtyphotonics.com/capabilities/fiber_bragg_gratings.html 